\title{
0092-8240(95)00306-B
}

\section{EXTENDING THE QUASI-STEADY STA'TE APPROXIMATION BY CHANGING VARIABLES}

- JOSÉ A. M. BORGHANS* and ROB J. DE BOER

Theoretical Biology,

Utrecht University,

Padualaan 8,

$3584 \mathrm{CH}$ Utrecht,

The Netherlands

(E.mail: jbo@alive.biol.ruu.nl and rdb@alive.biol.ruu.nl)

- LEE A. SEGEL

Dept. of Applied Mathematics and Computer Science,

The Weizmann Institute of Science,

Rehovot 76100,

Israel

(E.mail:lee@wisdom.weizmann.ac.il)

The parameter domain for which the quasi-steady state assumption is valid can be considerably extended merely by a simple change of variable. This is demonstrated for a variety of biologically significant examples taken from enzyme kinetics, immunology and ecology.

1. Introduction. Prototypical in biochemistry is the reversible reaction between enzyme $E$ and substrate $S$ to give complex $C$, which irreversibly yields product $P$ :

$$
E+S \underset{k_{-1}}{\stackrel{k_{1}}{\longrightarrow}} C \stackrel{k_{2}}{\longrightarrow} E+P
$$

The classical Michaelis-Menten approximation for scheme (1a) is the archetypal example for the use of the quasi-steady state (QSS) approximation. This approximation is a major simplifying step throughout biology, with its enormous range of time scales, and indeed in many other branches of science. The quasi-steady state assumption (QSSA) often yields revealing analytic formulas and it frequently circumvents problems of stiffness in the numerical integration of systems of differential equations. It is thus of

\footnotetext{
*Author to whom all correspondence should be addressed.
} 
considerable utility to be able to characterize parameter domains wherein a QSSA provides a valid approximation.

Virtually all biochemistry texts discuss the application of the QSSA to (1a) and its consequences, such as the use of the Lineweaver-Burk plot to obtain the maximum velocity of the reaction $V_{\max }$ and the Michaelis constant $K_{m}$. As far as we know, none of these texts indicates conditions under which the QSSA should be valid. Such conditions do appear in the literature, but only rather recently has it been shown (Palsson, 1987; Segel, 1988; Segel and Slemrod, 1989) that the usually cited requirement $E_{0} \ll S_{0}$ is too strong; the classical QSSA is in fact valid providing that

$$
E_{0} \ll S_{0}+K_{m},
$$

where $K_{m}$ is the Michaelis constant. See the derivation of (11) below.

This paper examines scheme (1a) when there is an excess of enzyme, so that (1b) does not hold. The classical QSSA breaks down in these situations, which can be encountered in vivo (Straus and Goldstein, 1943, p. 581; Sols and Marco, 1970) or in biotechnological applications. Remarkably, a simple change of variable permits the validity of the classical QSSA to be considerably extended so that the new situations are covered in many instances.

We show that "enzyme excess" arises naturally in schemes of the form

$$
E+S \underset{k_{-1}}{\stackrel{k_{1}}{\longrightarrow}} C \stackrel{k_{2}}{\longrightarrow} E+2 S .
$$

One example of (2) is the proliferation of T cells in response to antigen. Then $S$ denotes a replicating T cell, $E$ a site on an antigen-presenting cell (APC) and $C$ a complex of a T cell bound to an APC. This scheme has been analyzed by De Boer and Perelson (1994) employing the classical Michaelis-Menten approach. We here improve upon this earlier analysis. Another instance of (2) is one wherein $E$ is a catalytic RNA molecule while $S$ is another RNA molecule whose replication is catalyzed by $E$ (Eigen and Schuster, 1979).

A major point of the present paper is to show that, paradoxically, the standard QSSA for the complex $C$ in fact remains valid in a parameter domain that overlaps (1b), but also considerably extends (1b), provided merely that the free substrate concentration $S$ is replaced by the total substrate concentration $\bar{S} \equiv S+C$. In pursuing this goal, we employ and extend earlier considerations concerning the validity of the QSSA. Thus this paper also serves as a brief and up to date primer and case study on how to estimate when the QSSA is applicable.

After discussing the "total QSSA" for the prototype example (1a), we present several models where the same basic idea leads to useful new 
approximate solutions: models for replication schemes such as (2) and for certain predator-prey interactions.

2. The Standard QSSA and its Limits of Validity. Differential equations corresponding to (1a) can be written as

$$
\begin{aligned}
& \frac{\mathrm{d} S}{\mathrm{~d} t}=-k_{1}\left(E_{0}-C\right) S+k_{-1} C, \\
& \frac{\mathrm{d} C}{\mathrm{~d} t}=k_{1}\left[\left(E_{0}-C\right) S-K_{m} C\right],
\end{aligned}
$$

where

$$
K_{m}=\frac{k_{-1}+k_{2}}{k_{1}} .
$$

The conservation law

$$
E+C=E_{0}
$$

yields $E$. Initial conditions are

$$
\begin{array}{ll}
S(0)=S_{0}, & C(0)=0, \\
E(0)=E_{0}, & P(0)=0 .
\end{array}
$$

(Note that the choice $P(0)=0$ does not influence $S, E$ or $C$.)

In the standard approach, one assumes that after a fast transient, $C$ can be regarded as in equilibrium. From $\mathrm{d} C / \mathrm{d} t=0$ it follows that

$$
C=\frac{E_{0} S}{K_{m}+S} .
$$

A differential equation for $S$, valid after the transient, can be most easily derived by realizing that if $\mathrm{d} C / \mathrm{d} t$ is effectively zero, then (3b) can be added to (3a), yielding

$$
\frac{\mathrm{d} S}{\mathrm{~d} t}=-k_{2} C,
$$


i.e.

$$
\frac{\mathrm{d} S}{\mathrm{~d} t}=-\frac{k_{2} E_{0} S}{K_{m}+S} .
$$

It is conventional to assume that the substrate level changes negligibly during the fast transient so that ( $5 \mathrm{~b}$ ) can also serve as an "initial condition" for (7b). Differential equation ( $7 \mathrm{~b}$ ) and initial condition (5b) thus constitute the QSSA. One hopes that this QSSA will provide a good approximation for calculating the post-transient development of the system under consideration.

According to the procedure described by Segel (1988), the first step in attempting to determine the parameter ranges for which the QSSA is valid is to estimate two time scales. These are $t_{C}$, the time that characterizes the duration of the fast transient, and $t_{S}$, the magnitude of time required for a significant change in $S$ during the post-transient period. To estimate the fast time scale $t_{C}$ we model the initial rapid accumulation of $C$ by substituting $S=S_{0}$ in equation (3b). This transforms equation (3b) into a linear equation of which

$$
t_{C}=\frac{1}{k_{1}\left(S_{0}+K_{m}\right)}
$$

is the time scale. Another way to obtain the same estimate of $t_{C}$ is to realize (from (6) with $S=S_{0}$ ) that during the fast transient the total change of the complex is approximately $\Delta C=E_{0} S_{0} /\left(K_{\mathrm{m}}+S_{0}\right)$. At the maximum rate at which the complex increases (see equation (3b)) this indeed takes $t_{C}=\Delta C /\left(k_{1} E_{0} S_{0}\right)$ time units.

To estimate the slow (substrate) time scale $t_{S}$ we consider $\mathrm{d} S / \mathrm{d} t$ after the fast transient, i.e. equation (7b). We employ the characterization of $t_{S}$ that was suggested by Segel $(1984$, p. 56), i.e. we take the maximum post-transient change in substrate, $S_{0}$, divided by the post-transient maximum of $|\mathrm{d} S / \mathrm{d} t|$, obtained by substituting $S=S_{0}$ in (7b). Thus we write

$$
t_{s}=\frac{S_{0}}{|\mathrm{~d} S / \mathrm{d} t|_{\max }},
$$

i.e.

$$
t_{S}=\frac{K_{m}+S_{0}}{k_{2} E_{0}}
$$


The first condition necessary for the QSSA is that the fast time scale $t_{C}$ is indeed much smaller than the slow time scale $t_{s}$. This yields

$$
\frac{k_{2} E_{0}}{k_{1}\left(S_{0}+K_{m}\right)^{2}} \ll 1 \text {. }
$$

Secondly, to insure that equation (5b) can be taken as an "initial condition," we require that there be a small fractional depletion of substrate during the initial transient. This is ensured by demanding that the fractional change $\left|\Delta S / S_{0}\right|$ is small during the fast transient. We overestimate $\Delta S$ by the product of the maximum rate of depletion of $S$, i.e. (from equation (3a)) $k_{1} E_{0} S_{0}$, with the duration of the fast transient $t_{C}$. From this we find that the condition $\left|\Delta S / S_{0}\right| \ll 1$ requires

$$
\frac{E_{0}}{S_{0}+K_{m}} \ll 1
$$

Since (10) can be written

$$
\frac{E_{0}}{S_{0}+K_{m}} \frac{1}{1+\left(k_{-1} / k_{2}\right)+\left(S_{0} k_{1} / k_{2}\right)} \ll 1
$$

we see that condition (11) is stronger than condition (10). Thus, (11) guarantees the accuracy of the classical OSSA.

2.1. The effects of replacing free by total substrate concentration. In conditions of enzyme excess, when (11) and hence the standard QSSA are not expected to be valid we introduce the total substrate concentration $\bar{S}$, where

$$
\bar{S} \equiv S+C .
$$

$\bar{S}$ rather than $S$ will now be our substrate variable. The total substrate $\bar{S}$ cannot be depleted by the formation of complex. Because the validity of the classical QSSA depends strongly on negligible initial depletion of substrate (see the derivation of equation (11)), this simple variable change is expected to have an important effect. We derive the conditions for the QSSA of this redefined "total substrate" model, which we term the tQSSA.

If we substitute $\bar{S}$ for $S$ the governing equations become

$$
\begin{aligned}
& \frac{\mathrm{d} \bar{S}}{\mathrm{~d} t}=-k_{2} C, \\
& \frac{\mathrm{d} C}{\mathrm{~d} t}=k_{1}\left[\left(E_{0}-C\right)(\bar{S}-C)-K_{m} C\right],
\end{aligned}
$$


with initial conditions

$$
\bar{S}(0)=S_{0}, \quad C(0)=0 .
$$

Assuming $\mathrm{d} C / \mathrm{d} t=0$ as bcforc, but now using (13b), we find that $C$ should be replaced by a solution of the quadratic

$$
C^{2}-\left(E_{0}+K_{m}+\bar{S}\right) C+E_{0} \bar{S}=0 .
$$

The constraint $C \leq E_{0}$, which follows from (5a) and the non-negativity of $E$, implies that (15) has a unique solution. (One takes the negative square root in the quadratic formula.)

Quadratics such as (15) are common in kinetic calculations. For use in further kinetic manipulations, it is helpful to replace the somewhat complicated analytic formula for the solution of the quadratic by a simpler and more transparent expression. (This step is not essential to our main line of argument.) Such an expression is provided by a two-point Padé approximant (Baker and Graves-Morris, 1984). The lowest order Padé approximant consists of the quotient of two linear functions of $\bar{S}$. Around $\bar{S}=0$ and for $\bar{S} \rightarrow \infty$ we approximate equation (15) by

$$
C=\frac{E_{0} \bar{S}}{E_{0}+K_{m}} \quad \text { and } \quad C=E_{0}
$$

respectively. Thus the Padé approximant is

$$
C=\frac{E_{0} \bar{S}}{E_{0}+K_{m}+\bar{S}} .
$$

Formula (17) can also be obtained by neglecting the $C^{2}$ term in (15). This is consistent if for any value of $\bar{S}$,

$$
C=\frac{E_{0} \bar{S}}{E_{0}+K_{m}+\bar{S}} \ll E_{0}+K_{m}+\bar{S},
$$

i.e. if for any value of $\bar{S}$,

$$
1 \ll\left(1+\frac{\bar{S}}{E_{0}}+\frac{K_{m}}{E_{0}}\right)\left(1+\frac{E_{0}}{\bar{S}}+\frac{K_{m}}{\bar{S}}\right) .
$$


This certainly holds if $\bar{S}$ is either large or small compared to $E_{0}$. Even if $\bar{S}=E_{0}$, the right side of (19) is not less than $\left(2+K_{m} / E_{0}\right)^{2}$. Thus we conclude that neglecting the $C^{2}$ term in (15) to obtain (17) is indeed justified. Cha and Cha (1965) and Cha (1970) developed another method for deriving equation (17) as an approximation of (15). Cha (1970) numerically shows that the approximation is generally good.

To obtain an estimate for the range of validity of the new tQSSA, let us first estimate the time scale for the fast transient. During this period the complex concentration $C$ begins from an initial value of zero and remains relatively small. Thus, again neglecting the terms quadratic in $C$ when adapting (13b) for our present purposes, we obtain

$$
\frac{\mathrm{d} C}{\mathrm{~d} t}=k_{1}\left[E_{0} S_{0}-\left(E_{0}+S_{0}+K_{m}\right) C\right]
$$

In (20) we have made the simplifying approximation, which should be well warranted during the brief transient, that $\bar{S}(t) \approx S_{0}$. From the solution of (20) it is clear that

$$
t_{C}=\frac{1}{k_{1}\left(E_{0}+S_{0}+K_{m}\right)} .
$$

Note that $t_{C}$ can again be derived by calculating $\Delta C /|\mathrm{d} C / \mathrm{d} t|_{\max }$ as outlined just below equation (8).

It is at first surprising that the time scale $t_{C}$ in (21) is not the same as its counterpart in (8). The " $\Delta C$ " approach to calculating $t_{C}$ leads to the realization that the different values of $t_{C}$ can be traced to the different expressions of (6) and (17) for $C$. However, for (6) to be valid then it is necessary that $E_{0} \ll S_{0}+K_{m}$ (condition (11)). If this is the case, then the $E_{0}$ term is negligible in (17), at least until the considerable time elapses for $\bar{S}$ to drop below $S_{0}$. Additionally, if the $E_{0}$ term if negligible, the alternative formulas for $C$ and for $t_{C}$ are in fact identical. Thus whenever the alternatives (8) and (6) of the classical QSSA are valid they give answcrs that are indistinguishable from the tQSSA counterparts (21) and (17); only when (8) and (6) are invalid are they truly different from (21) and (17).

In order to estimate the slow time scale $t_{\bar{S}}$ we again consider the maximum change of $\bar{S}$ divided by the maximum rate of change of total substrate after the fast transient. From equation (13a) with $C$ given by the Padé approximant of equation (17), and with $\bar{S}=S_{0}$, calculation of 
$S_{0} / \mathrm{d} \bar{S} /\left.\mathrm{d} t\right|_{\max }$ yields

$$
t_{\bar{S}}=\frac{E_{0}+S_{0}+K_{m}}{k_{2} E_{0}} .
$$

The necessary condition for the validity of the tQSSA, $t_{C} \ll t_{\bar{S}}$, thus takes the following form:

$$
\frac{k_{2} E_{0}}{k_{1}\left(E_{0}+S_{0}+K_{m}\right)^{2}} \ll 1 .
$$

To check that initial condition (14a) is appropriate for our tQSSA, we require that the change of $\bar{S}$ is small during the fast transient. Paralleling the derivation of (11), we multiply the maximal value of $|\mathrm{d} \bar{S} / \mathrm{d} t|$ with the duration $t_{C}$ of the transient. By equation (13a) this maximum rate of change is $k_{2} C_{\max }$. An upper limit for $C_{\max }$ during the fast transient is the QSS value that the complex is approaching. Employing the Padé approximant (equation (17)), and substituting $\bar{S}=S_{0}$ (which is also an overestimate), we estimate $C_{\max }$. With this we find that (23) is also the condition that ensures that $\left|\Delta \bar{S} / S_{0}\right|$ is small.

Thus condition (23) suffices for the validity of the tOSSA. Below we confirm numerically that if equation (23) is not satisfied we indeed find that (i) after the fast transient, trajectories fail to correspond to the QSSA and also that (ii) during the fast transient, total substrate is depleted by the formation of product.

Finally we observe that we can rewrite equation (23) in the form

$$
\left(1+\frac{E_{0}+S_{0}}{k_{2} / k_{1}}+\frac{k_{-1}}{k_{2}}\right)\left(1+\frac{S_{0}+K_{m}}{E_{0}}\right) \gg 1 .
$$

The left side of (24a) is always greater than unity, so that we expect that the QSSA will always be at least roughly valid. Moreover, there are several different conditions any one of which guarantees that (24a) holds. These are

$$
E_{0}+S_{0} \gg k_{2} / k_{1}, \quad k_{-1} \gg k_{2}, \quad S_{0}+k_{m} \gg E_{0} .
$$

Note from (24d) the important finding that when the standard QSSA is valid, then so is the tQSSA. Thus it appears that our new approach considerably extends the parameter range for which a QSSA can be applied.

3. Comparing the Standard QSSA with the tQSSA. Conditions (11) and (23) can be plotted in parameter space to compare the regions of validity of 
the QSSA and the tQSSA. Note that this need not be a fair comparison because conditions (11) and (23) need not be equally strong for systems (3) and (13), respectively. Both conditions are of the form $f\left(E_{0}, S_{0}\right) \ll 1$. For definiteness, we draw in Fig. 1 the regions corresponding to $f\left(E_{0}, S_{0}\right) \leq 0.1$. For $k_{1}=10, k_{-1}=1$ and $k_{2}=0.1$ (i.e. $K_{m}=0.11$ ) the standard QSSA is valid in the dotted region in Fig. 1a. The tQSSA is valid for any initial condition, by $(24 \mathrm{c})$. Upon increasing the rate of product formation to $k_{2}=10$ (i.e. $K_{m}=1.1$ ), a relatively small curved region appears within which the tQSSA is not valid (see Fig. 1b; note the change in scale). The (dotted) validity region of the standard QSSA has remained almost the same. Thus Fig. 1 illustrates that when the standard QSSA is valid, the tQSSA is valid also. Additionally, the figure suggests that the tQSSA is valid for a much larger domain of initial conditions.

Generally, an analysis like that of Fig. 1 can best be performed in terms of dimensionless parameters. We nevertheless show the original parameters because the figure would remain qualitatively the same if we were to employ the following three dimensionless parameters:

$$
\eta=\frac{E_{0}}{K_{m}}, \quad \sigma=\frac{S_{0}}{K_{m}}, \quad \kappa=\frac{k_{2}}{k_{2}+k_{-1}} .
$$

The dotted and shaded regions in Fig. 1 would now, respectively, correspond to

$$
\frac{\eta}{1+\sigma} \leq 0.1 \text { and } \frac{\kappa \eta}{(\eta+\sigma+1)^{2}} \leq 0.1
$$

(a)

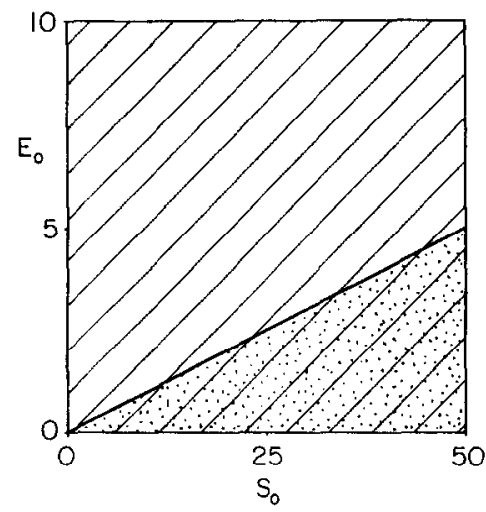

(b)

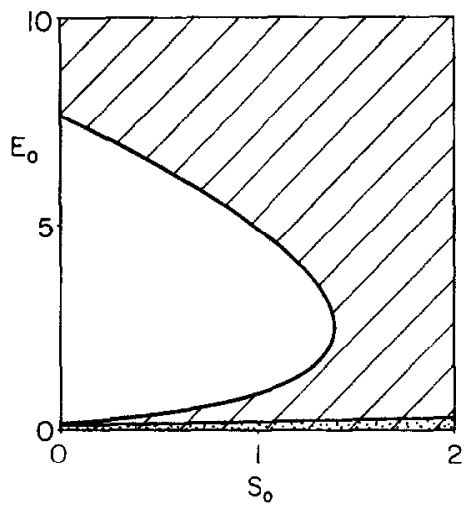

Figure 1. Validity of the QSS and the tQSS assumptions for the standard enzyme-substrate-complex scheme (1a), in the $E_{0}-S_{0}$ plane. Parameters: $k_{1}=$ 10 and $k_{-1}=1$, (a) $k_{2}=0.1\left(K_{m}=0.11\right)$; (b) $k_{2}=10\left(K_{m}=1.1\right)$. Dotted domain: QSSA valid; shaded domain: tQSSA valid. 
which have the same form as the curves in Fig. 1 when plotted as functions of $\eta$ and $\sigma$. (Increasing $k_{2}$ corresponds to increasing $\kappa$, which indeed decreases the region in which the tQSSA is valid.) We stress that the results of Fig. 1 provide representative examples of the ranges of validity of the QSSA and the tQSSA.

3.1. Numerical confirmation. In Fig. 2 we numerically compare the two QSS approximations in the $S-C$ and the $\bar{S}-C$ phase planes. The solutions of $\mathrm{d} C / \mathrm{d} t=0$, given by equation (6) and the valid root of (15), are shown as light lines in panels (a) and (b), respectively, for different values of $E_{0}$. The heavy lines represent exact solution trajectories for the corresponding values of $E_{0}$, with initial conditions $C(0)=0$ and $S(0)=\bar{S}(0)=0.1$. An important assumption for the QSS approximation is that the change in the substrate during the fast transient is small, i.e. the initial portion of the trajectory should be nearly vertical in the phase plane.

(a)

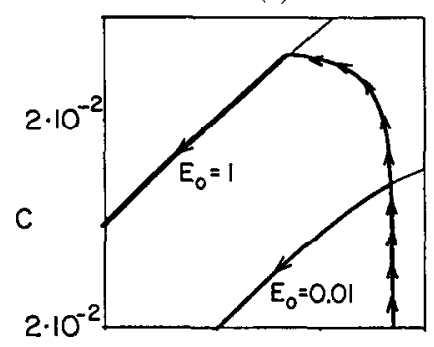

(c)

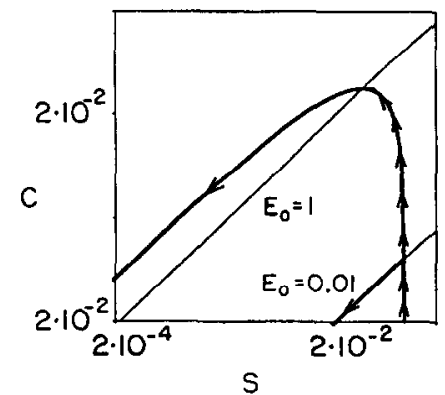

(b)

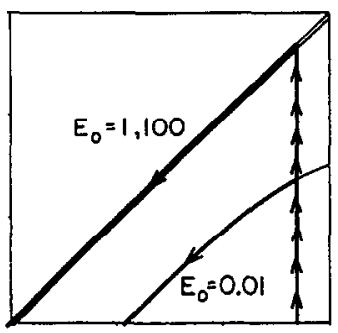

(d)

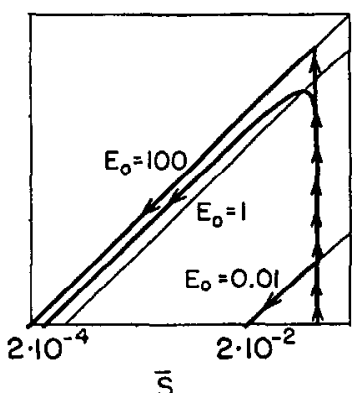

Figure 2. Trajectories approaching the QSS approximation of the product model (3)-(5) with (panels b, d) and without (panels a, c) change of variables from free substrate $S$ to total substrate $\bar{S}$. In the substrate versus complex phase plane the heavy lines depict numerically computed trajectories of the full model for initial conditions $C(0)=0$ and $S(0)=\bar{S}(0)=0.1$. The light curves depict the QSS equations for $C$, i.e. equation (6) or the solution of (15). The arrows indicate the direction of the trajectories: a high arrow density implies a relatively rapid traversal. Parameters: $k_{1}=10$ and $k_{-1}=1$. In panels $(\mathrm{a}, \mathrm{b}) k_{2}=0.1\left(K_{m}=0.11\right)$; in panels $\left(c\right.$, d) $k_{2}=10\left(K_{m}=1.1\right)$. 
On the basis of Fig. 1 we expect for $k_{2}=0.1$ that the standard QSSA will become invalid by increasing $E_{0}$ while the tQSSA will remain unaffected. This is confirmed by Fig. 2a, b. For $E_{0}=0.01$ the trajectory starts out rapidly and vertically and then sharply turns left to slowly follow the QSS solution in both (a) and (b). For $E_{0}=1$ the trajectory of the standard model in panel (a) depicts strong and rapid depletion of substrate before the relatively slow QSS solution is attained. This is not the case in panel (b), with the change of variables, even if $E_{0}=100$. Thus the tQSSA is valid, where the classical QSSA fails.

For $k_{2}=10$ the expectation is more challenging. The standard OSSA should simply lose validity by increasing $E_{0}$. The tQSSA however should become worse at intermediate values of $E_{0}$ but become accurate again at high values (by (24)). This is confirmed in Fig. 2c, d. As expected the behavior of the standard QSSA model in panel (c) is comparable to that in panel (a). Moreover, the trajectories of the tQSSA model indeed show the strongest substrate depletion when $E_{0}=1$. Additionally, when $E_{0}=1$ we see that after the fast transient the trajectory fails to approach the tQSS approximation. Remember that condition (23) pertains to both the difference in time scales and to the depletion of substrate.

4. The Reverse QSSA. There is an alternative approach to simplifying the governing equations (3), by assuming that $\mathrm{d} S / \mathrm{d} t=0$ rather than $\mathrm{d} C / \mathrm{d} t=0$. This approach, termed the reverse QSSA (rQSSA for short), has been outlined by Segel and Slemrod (1989). Like the tQSSA, the rQSSA is valid when $E_{0}$ is "large", in contrast to the validity condition " $E_{0}$ small" for the classical QSSA. In the Appendix we show that in the parameter range where the rQSSA is valid, our new and simpler tQSSA is valid also, and that both approximations give similar results.

5. The Replication Model. Initial conditions with large $E_{0}$ are typical of the replication scheme (2). The differential equations corresponding to (2) are

$$
\begin{aligned}
& \frac{\mathrm{d} S}{\mathrm{~d} t}=-k_{1}\left(E_{0}-C\right) S+\left(k_{-1}+2 k_{2}\right) C, \\
& \frac{\mathrm{d} C}{\mathrm{~d} t}=k_{1}\left[\left(E_{0}-C\right) S-K_{m} C\right],
\end{aligned}
$$

where (26b) is identical to (3b), and the conservation law and initial conditions are identical to equations $(5 \mathbf{a}-\mathrm{d})$. 
The standard QSSA approach for this replication model proceeds analogously to the analysis of equations (6-11). Thus, setting $\mathrm{d} C / \mathrm{d} t=0$ in $(26 \mathrm{~b})$, we obtain equation (6), which upon substitution into (26a) gives

$$
\frac{\mathrm{d} S}{\mathrm{~d} t}=k_{2} C
$$

i.e.

$$
\frac{\mathrm{d} S}{\mathrm{~d} t}=\frac{k_{2} E_{0} S}{K_{m}+S}
$$

$S(0)=S_{0}$ is assumed as an "initial condition." Note that in (27b) $S$ increases by replication, in contrast to (3) where $S$ decreases by transformation into product.

Because the differential equations of the complex, i.e. equations (26b) and ( $3 \mathrm{~b})$ are the same for the standard and replication models, the fast time scale $t_{C}$ is also the same and is hence given by equation (8). Upon contemplating the slow time scale $t_{S}$ we observe that equation (27b) is identical to (7b) except for a difference in sign. However, we can not employ the characterization ( $9 \mathrm{a}$ ) for $t_{s}$ because substrate now increases, i.e. the maximum change of substrate is not defined. Instead we propose a slightly modified estimate of $t_{S}$ : we calculate the relative change in substrate, i.e. $(\mathrm{d} S / \mathrm{d} t) / S$, right after the fast transient, i.e. when $S \approx S_{0}$. Thus, we consider equation (27) for $S(0)=S_{0}$, divide by $S_{0}$, and take the inverse to obtain $t_{S}$ given by equation (9). Note that this modified estimate also applies to the product model (3) and that both approaches give the same estimate for the slow time scale.

For consistency we require that the substrate is hardly depleted during the fast transient. From equation (26a) the maximum depletion rate is $k_{1} E_{0} S_{0}$ which maximally lasts $t_{C}$ time units. This again yields condition (11). Thus, we find equations (10) and (11) as the two conditions for the QSSA. We conclude that there is no difference in the QSSA conditions for the standard approaches to the product and replication models.

5.1. Replacing free by total substrate: the tQSSA. Defining $\bar{S} \equiv S+C$ as in equation (12) we write for scheme (2)

$$
\begin{aligned}
& \frac{\mathrm{d} \bar{S}}{\mathrm{~d} t}=k_{2} C, \\
& \frac{\mathrm{d} C}{\mathrm{~d} t}=k_{1}\left[\left(E_{0}-C\right)(\bar{S}-C)-K_{m} C\right],
\end{aligned}
$$


where the equation for $\mathrm{d} C / \mathrm{d} t$ is again identical to equation (13b) and the initial conditions are given by equations $(14 a, b)$.

Solving $\mathrm{d} C / \mathrm{d} t=0$ we find equation (15) and its Padé approximant equation (17). For calculating the fast time scale $t_{C}$ we employ equation (20) to obtain equation (21). For the slow time scale $t_{\bar{S}}$ we consider $\mathrm{d} \bar{S} / \mathrm{d} t$ right after the fast transient, i.e. we use equation (28a) with $C$ approximated by equation (17) and $\bar{S}=S_{0}$, to obtain equation (22) and hence equation (23). As before, equation (23) also guarantees the approximate initial condition (14a).

Our finding that the QSS approximation is extended by making a tQSSA is illustrated for the present example in Fig. 3. The light lines depict the same QSS solutions as those in Fig. 2a, b. The heavy lines again represent the exact trajectories for the same parameters and initial conditions. For $E_{0}=0.01$ the trajectory has the required nearly vertical initial behavior. For $E_{0}=1$ and $E_{0}=100$ the trajectories in panel (b) have the required initial behavior, whereas those in panel (a) do not.

Figure 4 illustrates in a different way the advantage of the total substrate formulation in the replication model. We plot the free and total substrate concentrations, $S$ and $\bar{S}$, as functions of time. Here F denotes the solution of the full model, i.e. equation (26) or (28), Q that of the QSSA model, i.e. equation (26a) with (6) or (28a) with the valid solution of (15) and $P$ that of the Padé approximation, i.e. equation (28a) with (17). When $E_{0}=0.01<S_{0}$ $=0.1$ the QSSA is valid in both models and all solutions are identical (not shown). If $E_{0}=100>S_{0}=0.1$ the two tQSS variants $\mathrm{Q}$ and $\mathrm{P}$ closely resemble the full solution $F$ (Fig. 4b). However, there is a large difference

(a)

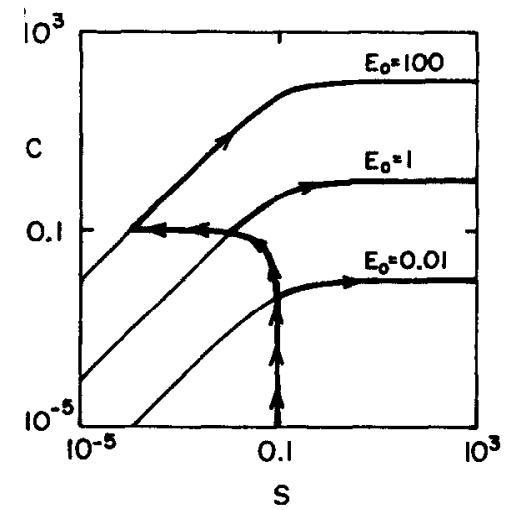

(b)

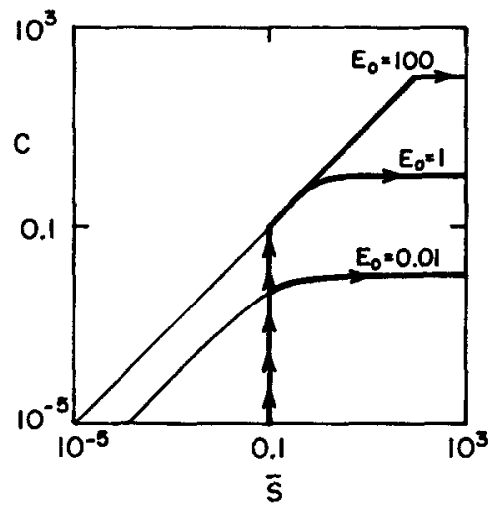

Figure 3. Similar to Fig. $2 a$ and $b$, but for the replication model (26) with $k_{2}=0.1$. The light QSS curves are the same as in Fig. 2 (but note different scales). Since now $\mathrm{d} S / \mathrm{d} t>0$, these curves are traced "backwards." 
(a)

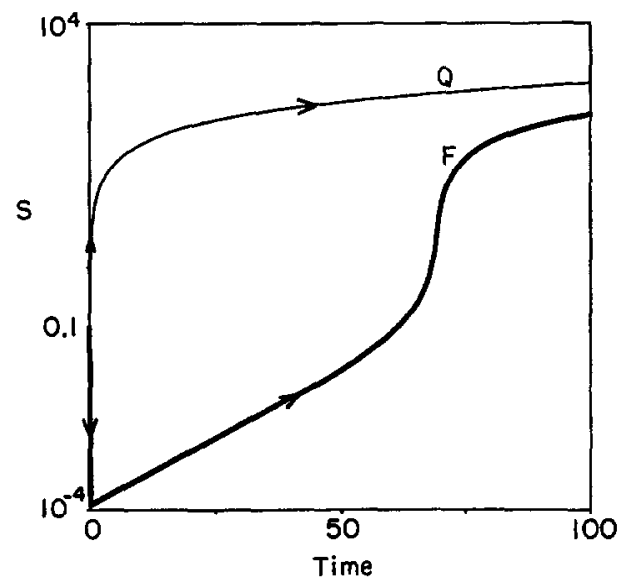

(b)

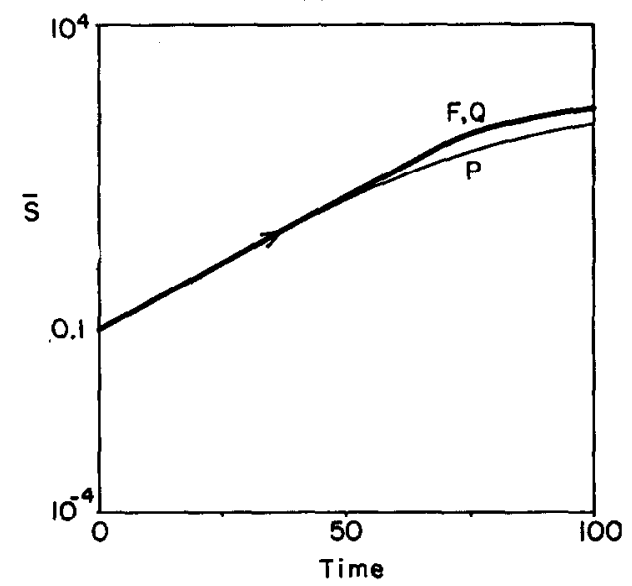

Figure 4. Time plots of free substrate concentration $S$ and total substrate concentration $\bar{S}$ for the replication model (26), for high concentrations of enzyme. The solution of the full model is marked as $F$, that of the quasi-steady state model as Q and that of the Padé approximation as P. Parameters are as in

Fig. 3. The initial conditions are $C(0)=0, S(0)=\bar{S}(0)=0.1$ and $E(0)=100$.

between $\mathrm{Q}$ and $\mathrm{F}$ in the classical model of Fig. 4a: the "approximation" $\mathrm{Q}$ incorrectly shows a rapid growth in free substrate $S$ while in fact $S$ initially decays rapidly, owing to complex formation, before replication causes rapid growth of $S$. This defect is absent when we change variables to $\bar{S}$ (see Fig. 4 b), for $\bar{S}$ is not depleted by complex formation.

6. T Cell Proliferation. De Boer and Perelson (1994) modelled T cell growth on the basis of schemes similar to scheme (2). They derived the equivalent of equation (27), added a source and decay term and proposed the following model of $T$ cell growth, where "substrate" now means the free T cell density $T$ :

$$
\frac{\mathrm{d} T}{\mathrm{~d} t}=\sigma+T\left(\frac{\rho A}{K+T}-1\right)
$$

Here $A$ is the concentration of sites presenting antigen and $\sigma$ is the source of naive $\mathrm{T}$ cells from the thymus. Equation (29) naturally implements competition between $\mathrm{T}$ cells for seeing antigen. The interesting implications of this model are discussed by De Boer and Perelson (1994). The main problem with this model is that it has an unbounded per capita rate of $\mathrm{T}$ cell growth. The growth rate of a $\mathrm{T}$ cell population increases without bound as $A \rightarrow \infty$. We are now able to solve this problem by changing variables to 
total T cells, $\bar{T} \equiv T+C$, and using equations (17) and (28) to write

$$
\frac{\mathrm{d} \bar{T}}{\mathrm{~d} t}=\sigma+\bar{T}\left(\frac{\rho A}{K+\bar{T}+A}-1\right) .
$$

Here $\rho$ represents the maximum proliferation rate for an individual $\mathrm{T}$ cell (which is formally achicved when $A \rightarrow \infty$ ). This model is much more realistic and also accounts for competition between $\mathrm{T}$ cells for seeing antigen.

A disadvantage of introducing $\bar{T}$ is the quadratic equation one has to solve (i.e. equation (15)), which, for instance, prevents one from repeating the analysis of De Boer and Perelson (1994) for $n>1 \mathrm{~T}$ cell populations seeing $m>1$ different antigens. De Boer and Perelson (1995) do derive a model for $n \mathrm{~T}$ cell populations interacting with one antigen.

7. Predator-Prey Interactions. The Lotka-Volterra model for a predator species feeding upon a prey species is classical in ecology. A general model for the interaction between a prey $X$ and predator $Y$ is

$$
\begin{aligned}
& \frac{\mathrm{d} X}{\mathrm{~d} t}=X(1-X)-a f(X, Y), \\
& \frac{\mathrm{d} Y}{\mathrm{~d} t}=a b f(X, Y)-c Y
\end{aligned}
$$

where

$$
f(X, Y)=X Y \text { or } f(X, Y)=\frac{X Y}{K+X}
$$

Equation (32b) is the Holling type II response. Since in (32b) $f(X, Y) \propto Y$ when $X \gg K$, the parameter $a$ is interpreted as the maximum number of prey eaten per predator per unit time.

Employing the approach outlined above we can formally derive a generalized interaction term $F(X, Y)$ from the scheme

$$
X+Y \underset{k_{-1}}{\stackrel{k_{1}}{\longrightarrow}} C \stackrel{k_{2}}{\longrightarrow}(1+b) Y,
$$

where $b$ is the growth rate of the predator due to eating prey. Changing variables to total prey $\bar{X} \equiv C+X$ and total parameters $\bar{Y} \equiv C+Y$ and 
making a QSSA for $C$, we obtain

$$
\frac{\mathrm{d} C}{\mathrm{~d} t}=k_{1}\left[(\bar{X}-C)(\bar{Y}-C)-K_{m} C\right]=0,
$$

or

$$
C \approx \frac{\bar{X} \bar{Y}}{K_{m}+\bar{X}+\bar{Y}} .
$$

In addition

$$
\frac{\mathrm{d} \bar{X}}{\mathrm{~d} t}=-k_{2} C \text { and } \frac{\mathrm{d} \bar{Y}}{\mathrm{~d} t}=b k_{2} C
$$

Writing $k_{2}=a$ and $K_{m}=K$ we thus obtain

$$
F(\bar{X}, \bar{Y})=\frac{\bar{X} \bar{Y}}{K+\bar{X}+\bar{Y}}
$$

as a general interaction function. Because this saturates as a function of $\bar{X}$, the parameter $a$ in equation (31) has the same interpretation as it has in the Holling type II response.

Having a general function, we observe that the Holling type II response is retrieved when we assume that there is an excess of prey, i.e. $\bar{X} \gg \bar{Y}$, so that from (34b) $\bar{X} \gg C$. Equation (34a) then simplifies into

$$
\frac{\mathrm{d} C}{\mathrm{~d} t}=k_{1}\left[\bar{X}(\bar{Y}-C)-K_{m} C\right]=0 \quad \text { giving } \quad F(\bar{X}, \bar{Y})=\frac{\bar{X} \bar{Y}}{K+\bar{X}}
$$

Similarly, assuming an excess of predators, i.e. $\bar{Y} \simeq Y$, equation (34a) simplifies into

$$
\frac{\mathrm{d} C}{\mathrm{~d} t}=k_{1}\left[\bar{Y}(\bar{X}-C)-K_{m} C\right]=0 \quad \text { giving } \quad F(\bar{X}, \bar{Y})=\frac{\bar{X} \bar{Y}}{K+Y},
$$

which allows for interference between predators. The parameter $a$ however loses the above interpretation.

Our general function (36) has been proposed previously on intuitive grounds by DeAngelis et al. (1975) and Beddington (1975). The fact that this function can now be formally derived supports its usage in ecological 
models and provides clues and/or precautions for how to generalize (36) for a system with several predator and prey species (see De Boer and Perelson, 1995). Functions like this have also been proposed in the context of ratio-dependent predator-prey interactions (Arditi and Ginzberg, 1989). We think equation (36) is appropriate in this context because for $X \gg K$ it resembles the Holling function, with its maximum rate of predation per predator, whereas for $X \ll Y$ it allows for the interference between predators that is characteristic of models with ratio-dependent predation.

8. Summary and Discussion. Rather remarkably, there is still more to say about the standard enzyme-substrate-complex scheme (1a), with its mathematical formulation (3)-(5). In the classical approach, the concentrations of free substrate $S$ and the complex $C$ are taken as dependent variables. The quasi-steady state assumption (QSSA) $\mathrm{d} C / \mathrm{d} t=0$ is made, resulting in equation (6) for $C$ as a function of $S$ and hence differential equation (7b) for $\mathrm{d} S / \mathrm{d} t$. It is assumed that little substrate is consumed during the initial transient period before the QSSA is valid, so that $S(0)=S_{0}$ is taken as the initial condition for (7b). The validity of this procedure is assured if (11) holds.

We have explored a different approach, where the total substrate concentration $\bar{S}$ is employed instead of $S$. Again the assumption $\mathrm{d} C / \mathrm{d} t=0$ is made, but now this results in the quadratic equation (15) for $C$ as a function of $\bar{S}$. We show that (17) offers an accurate Padé approximation for (15), which provides an explicit formula for $C$. An equation for $\mathrm{d} \bar{S} / \mathrm{d} t$ can now be written, which is solved subject to the initial condition $\bar{S}(0)=S_{0}$. The validity of this total QSSA (tQSSA) is assured by (23).

By changing variables, from free substrate $S$ to total substrate $\bar{S}$ we have enlarged the domain of parameters for which it is permissible to employ the classical QSSA, $\mathrm{d} C / \mathrm{d} t=0$. It might be objected that this is getting something for nothing, for how can the same assumption be rendered more acceptable merely by a simple change of variables? However, recall (see Segel, 1988) that the essential reason why the QSSA holds is that the QSS variable (here $C$ ) has a fast intrinsic rate of change compared to the "non-QSS variable" (here $S$ ). For the parameter range of interest, our new "non-QSS variable" $\bar{S}$, the total substrate concentration, changes very much more slowly than $S$, and hence our change of variable should indeed lead to an improved approximation.

The classical QSSA looses its validity when condition (11) fails, i.e. when there is little substrate compared to enzyme. The allegedly slow (substrate) variable then becomes fast due to rapid formation of complex. This effect, 
which is particularly important during the initial transient, is evaded by the change of variables to total substrate, because total substrate can only change by the formation of product. This explains why $k_{2}$ appears in conditions (23) and (24).

When checking the validity of the classical QSSA, for example, we ascertained that the phase plane curve $C=C(S)$ of (6) was indeed approached by the numerical solution of the governing equations. This check also indicates that the graphs for $S(t)$ and $C(t)$ that are derived from the QSSA will be close to the correct values. The reason is that if indeed (6) holds, then $(7 \mathrm{~b})$ is a good approximation to the true equation for $\mathrm{d} S / \mathrm{d} t$. Hence the solution of ( $7 \mathrm{~b}$ ) should yield a good approximation, provided that the basic problem is not ill conditioned (so that a small error in the problem can lead to a large error in the solution) and provided that integration has not been carried out so long that even large errors have had a chance to accumulate.

For definiteness, in this paragraph we continue to discuss general matters in the framework of the classical QSSA. We have stressed that justification of the QSSA requires demonstrating two things, for the parameter domain in question. These are (i) that after a fast transient, one can approximate the $\mathrm{d} C / \mathrm{d} t$ equation by regarding it at steady state, yielding $C=C(S)$, and (ii) that the true initial condition $S=S_{0}$ remains approximately true after the transient. The path to demonstrating (ii) was transparent-estimate the change in $S$ during the transient. It is not clear, however, that our checking whether $t_{C} \ll t_{S}$ is the right way to demonstrate (i). (Although it is clear that consistency demands that the duration of the fast transient is relatively brief.) It appears that the best way to show that $t_{C} \ll t_{S}$ ensures that $C=C(S)$ is to use $t_{C}$ and $t_{S}$ in a formal adoption of suitable scaled variables, as described by Segel and Slemrod (1989). These authors show that $C=C(S)$ emerges from a singular perturbation analysis where the small parameter is the ratio $t_{C} / t_{S}$.

The change of variables into total substrate may give rise to complications in situations where the substrate also reacts with other variables. In the standard model one may assume that free substrate reacts with another variable but substrate in complex does not. Making the change of variables one typically writes that the total substrate variable, and hence both free substrate and complex, reacts with other variables. In the context of immunology, for an example of a $\mathrm{T}$ cell population interacting with both antigen and another (regulator) $\mathrm{T}$ cell population, see Borghans and De Boer (1995).

When the work reported here was essentially completed, we learned that there were antecedents for part of it. With precedent from the careful studies of Straus and Goldstein (1943) and Goldstein (1944) on 
enzyme-substrate-inhibitor systems, Reiner (1969), Cha and Cha (1965) and Cha (1970) used total substrate concentration in pursuing the implications of the QSS assumption $\mathrm{d} C / \mathrm{d} t=0$. They thus employed (15) to determine $C$ as a function of $\bar{S}$. They discussed different approximations of (15) in different parameter domains, but they never challenged the basic assumption $\mathrm{d} C / \mathrm{d} t=0$. That this assumption could well be inappropriate was recognized by Lim (1973), who illustrated his assertion by a numerical example.

Novel is delineation of the domains of validity of the various approximations, our central goal. Perhaps it should go without saying that the approach taken here can also be applied, at least in principle, to the many other kinetic equations where some type of QSSA could be appropriate.

We thank the non-linear systems (NLS) program of the Netherlands Organization for Scientific Research (NWO) for support of the visit of LAS to Utrecht. LAS acknowledges partial support by the U.S.-Israel Binational Science Foundation (Grant 92-00171).

\section{APPENDIX}

The Reverse QSSA. We here present the reverse QSSA (rQSSA for short) of Segel and Slemrod (1989) in a somewhat altered and extended form, since we wish to compare its results with those obtained above. Upon setting $\mathrm{d} S / \mathrm{d} t=0$ in (3a) we obtain

$$
S=\frac{K C}{E_{0}-C}
$$

where

$$
K=k_{-1} / k_{1}
$$

Substitution of (A1) into (3b) yields

$$
\frac{\mathrm{d} C}{\mathrm{~d} t}=-k_{2} C .
$$

(Another way to obtain (A3) is to add (3a) to (3b) after setting $\mathrm{d} S / \mathrm{d} t=0$ ) The complex concentration $C$ typically decreases substantially during the transient, but nonetheless an appropriate initial condition for (A3) can be derived (Segel and Slemrod, 1989).

A necessary condition for the validity of the rQSSA is that the time scale $t_{C}$ for post-transient changes in $C$ is long compared to the time scale $t_{S}$ for the rapid transient changes in $S$ that occur before (A1) is satisfied. From (A3), $t_{C}=k_{2}^{-1}$. To estimate $t_{S}$ we begin by employing (12) to replace $S$ by $\bar{S}-C$ in (A1). Rearranging the resulting equation we obtain

$$
C^{2}-\left(E_{0}+\bar{S}+K\right) C+E_{0} \bar{S}=0
$$


Equation (A4) differs from equation (15) only in that $K$ appears in (A4), where $K_{m}$ appears in (15). Given that $\bar{S}$ remains close to $S_{0}$ during the transient, we can replace $K_{m}$ by $K$ in the Padé formula (17) to estimate that at the end of the transient, $C$ has the value $\tilde{C}$, where

$$
\tilde{C}=\frac{E_{0} S_{0}}{E_{0}+K+S_{0}} .
$$

$C(0)=\tilde{C}$ is the appropriate "initial condition" for (A3).

Replacing $C$ by $\tilde{C}$ in (3a) yields

$$
\frac{\mathrm{d} S}{\mathrm{~d} t}=-k_{1}\left(E_{0}-\tilde{C}\right) S+k_{-1} \tilde{C} .
$$

It follows that $\left[k_{1}\left(E_{0}-\tilde{C}\right)\right]^{-1}$ provides an estimate of $t_{S}$, i.e.

$$
t_{s} \approx \frac{K+E_{0}+S_{0}}{k_{1} E_{0}\left(K+E_{0}\right)}
$$

This estimate is conservative ( $t_{S}$ is slightly overestimated). From the condition $t_{S} \ll t_{C}$, we thereby obtain the following conservative estimate for the parameter domain wherein the rQSSA is valid:

$$
\frac{k_{2}}{k_{1}} \ll \frac{\left(K+E_{0}\right) E_{0}}{K+E_{0}+S_{0}} .
$$

By comparison, condition (23) for the validity of the "total" or tQSSA can be written

$$
\frac{k_{2}}{k_{1}} \ll \frac{\left(K_{m}+E_{0}+S_{0}\right)^{2}}{E_{0}}
$$

The tQSSA is valid in a strictly larger parameter domain than the rQSSA because

$$
\frac{\left(K_{m}+E_{0}+S_{0}\right)^{2}}{E_{0}}>\frac{\left(K+E_{0}+S_{0}\right)^{2}}{E_{0}}>\frac{\left(K+E_{0}\right) E_{0}}{K+E_{0}+S_{0}}
$$

When both the tQSSA and the rQSSA are valid, that is when (A8) holds, consistency demands that both approximations be virtually identical. Comparing (A4) and (15), we see that this will be the case if

$$
E_{0}+\bar{S}+K_{m} \approx E_{0}+\bar{S}+K
$$

or

$$
k_{1} E_{0}+k_{1} \bar{S}+k_{2}+k_{-1} \approx k_{1} E_{0}+k_{1} \bar{S}+k_{-1}
$$


However, (A12) indeed holds, since (A8) implies that $k_{2} \ll k_{1} E_{0}$. Thus the tQSSA is to be preferred because it has a wider range of validity than the rQSSA and it does not require special derivation of a post-transient "initial condition."

\section{LITERATURE}

Arditi, R. and L. R. Ginzberg. 1989. Coupling in predator-prey dynamics: ratio-dependence. J. Theor. Biol. 139, 311-326.

Baker, G. A. and P. R. Graves-Morris. 1984. Padé approximants. Encyclopedia of Mathematics and its Applications. New York: Cambridge University Press.

Beddington, J. R. 1975. Mutual interference between parasites or predators and its effects on searching efficiency. J. Anim. Ecol. 51, 597-624.

Borghans, J. A. M. and R. J. De Boer. 1995. A minimal model for T-cell vaccination. Proc. R. Soc. London Ser. B 259, 173-178.

Cha, S. 1970. Kinetic behavior at high enzyme concentrations. J. Biol. Chem. 245, 4814-4818.

Cha, S. and C.-J. M. Cha. 1965. Kinetics of cyclic enzyme systems. Mol. Pharmacol. 1, $178-189$.

DeAngelis, D. L., R. A. Goldstein and R. V. O'Neill. 1975. A model for trophic interaction. Ecology 6, 881-892.

De Boer, R. J. and A. S. Perelson. 1994. T cell repertoires and competitive exclusion. J. Theor. Biol. 169, 375-390.

De Boer, R. J. and A. S. Perelson. 1995. Towards a general function for T cell proliferation. $J$. Theor. Biol. in press.

Eigen, M. and P. Schuster. 1979. The Hypercycle: A Principle of Natural Self-Organization. Berlin: Springer.

Goldstein, A. 1944. The mechanism of enzyme-inhibitor-substrate reactions. J. Gen. Physiol. 27, 529-580.

Lim, H. C. 1973. On kinetic behavior at high enzyme concentrations. AIChEJ 19, 659-661.

Palsson, B. O. 1987. On the dynamics of the reversible Michaelis-Menten reaction mechanisms. Chem. Eng. Sci. 42, 447-458.

Reiner, J. M. 1969. The Behavior of Enzyme Systems. New York: Van Nostrand Reinhold.

Segel, L. A. 1984. Modeling Dynamic Phenomena in Molecular and Cellular Biology. New York: Cambridge University Press.

Segel, L. A. 1988. On the validity of the steady state assumption of enzyme kinetics. Bull. Math. Biol. 6, 579-593.

Segel, L. A. and M. Slemrod. 1989. The quasi-steady state assumption: a case study in perturbation. SIAM Rev. 31, 446-477.

Sols, A. and R. Marco. 1970. Concentration of metabolites and binding sites. Implications in metabolic regulation. In Current Topics in Cellular Regulation, Vol. 2. New York: Academic Press.

Straus, O. H. and A. Goldstein. 1943. Zone behavior of enzymes. J. Gen. Physiol. 26, $559-585$. 